\title{
Reduction of Routing Overhead in Wireless Sensor Network with Modified AODV-TP (Constant Packet Size)
}

\author{
Rubaina Takhi \\ Student \\ Department of Electronics Technology \\ Guru Nanak Dev University Amritsar \\ Punjab-143005, India
}

\begin{abstract}
Power management is essential in Wireless Sensor Network because the nodes are very small in size having a small battery and that too of limited life. The routing algorithms for it should satisfy the features of optimization of energy consumption. Ad hoc On Demand Distance Vector routing protocol has been modified, this algorithm has been analyzed named as modified AODV-TP (Threshold Power) and is highlighted in detail. The theme is that only those nodes are chosen as neighbors of nodes during RREQ (Route Request) phase that are directed towards destination. Also, threshold is set for all the nodes. If the power of the node is less than threshold, it will not acknowledge Route Reply (RREP) packet. The alternate route with second lowest minimum distance will be selected to forward the packet. The comparison is done between two protocols in terms of routing overhead and average remaining energy of nodes and delay with variation in packet. The proposed algorithm turns out to be better in terms of power conservation.
\end{abstract}

\section{Keywords}

Wireless sensor network; power management; nodes; AODV

\section{INTRODUCTION}

Wireless Sensor Network consists of number of nodes which integrates processing, sensing and communication subsystem. These networks have variety of applications including habitat [1], heath [2], pipeline [3], environment monitoring, agriculture [4], food safety, smart living and disaster management. The nodes operate with batteries and these nodes are very small in size to accommodate large batteries. Also, it is not desirable to manually replace or recharge batteries. An efficient use of energy is a crucial concern in wireless sensor networks.

This paper enhances ad hoc on demand distance vector (AODV), which is one of the widely used reactive routing protocols, in order to reduce energy consumption and achieve the reliability of WSN [5]. In this proposed algorithm modified AODV-TP, the broadcast of RREQ (Route Request) packet has been modified. Only those nodes are chosen as neighbors of nodes during RREQ (Route Request) phase that are directed towards destination. Also, threshold value of each node is set; when the destination node replies back with respect to RREQ (Route request) packet each node acknowledges the RREP packet received. If the battery power of any node is less than threshold value it will not acknowledge the received RREP packet and the packet will be forwarded through different route. In AODV-TP, only RREP phase is modified as described above. There is no change in RREQ phase. RREQ packets are forwarded to all neighbors.

\section{RELATED WORK}

The conventional on demand routing algorithm like DSR (Dynamic Source Routing), AODV lead to quick depletion of the battery energy of the nodes because these protocols establish connections between nodes through the shortest path routes but are unaware of energy of nodes. The various attempts have been made to modify AODV by adding a number of parameters to AODV that balances the energy of nodes. The transmission power of the nodes is also varied according to the distance between the nodes.

Marina and Samir proposed Energy aware routing in Ad Hoc Networks in 2001 in which AODVEA selects a route with largest minimum residual energy and AODVM selects a route with the largest minimum residual energy and less hop count. There is no need to restart Route discovery if one of the links fails. But, there is need to uniquely identify each disjoint path on an end to end basis and to resolve issues related to ondemand multipath routing. [6]. Seema, Pinki and Rekha proposed AOMDV which also varies the transmission power between two nodes as per their distance [7]. It finds nodedisjoint paths by exploiting a particular property of flooding. Samundiswary and Anandkumar proposed EAODV protocol in 2012 which takes into account the residual energy of sensor nodes along with the hop count to avoid unbalanced energy consumption of sensor nodes [8]. Divya, Manisha and Hari proposed AODV-TP (threshold Power) for MANETs.

\section{SIMULATION ALGORITHM DESIGN}

\subsection{Problem Formulation}

As AODV considers simple hop count metric to select the best path, it is not suitable for WSN. Therefore, the main purpose of this paper is to consider not only hop count but also energy metric while selecting the best path.

The objective is to analyze, simulate and evaluate modified AODV-TP and to compare the performance of its power consumed, routing overhead and delay with AODV-TP.

\subsection{Proposed Routing Algorithm}

The source node initiates the RREQ (Route Request) packet and forwards that to its neighbors which in turn forward to their neighbors and so on until the destination node receives the RREQ packet. Only those nodes are chosen as neighbors of nodes during RREQ (Route Request) phase that are directed towards destination that is $\mathrm{x}$ as well as y coordinate of the neighbors should be greater than that of the corresponding coordinates of source. For example, in the figure N2 will be chosen as neighbor of N5 in RREP phase not N7. 


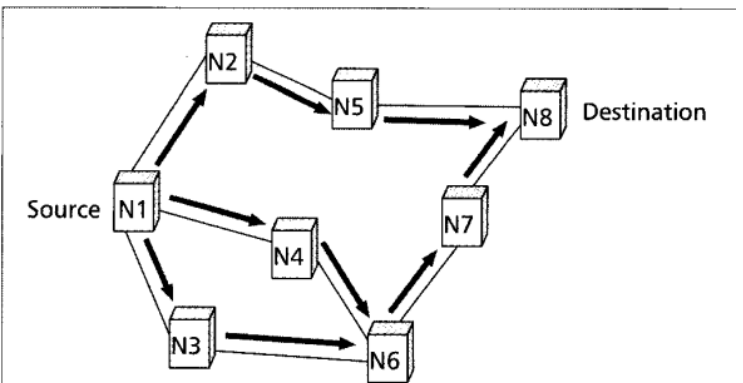

(a) Propagation of the RREQ

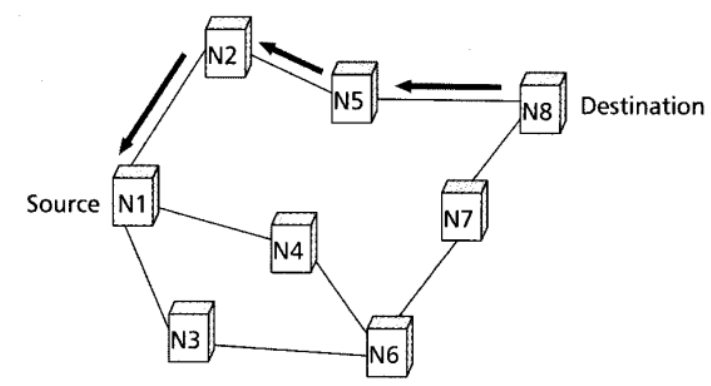

(b) Path of the RREP to the source

Fig 1: A wireless sensor network

RREP is broadcasted by the destination node. In AODV, sending RREP (Route Reply) from destination to source node is similar to transmission of RREQ packet. However this leads to more energy consumption. The algorithm for transmission of RREP packet for modified AODV-TP is illustrated below:-

1: Start

2: Set threshold value of energy (say $4 \mathrm{~J}$ ) for each node.

3: For each node do

4: Obtain distance measurements of all the neighbors using distance formula

5: Choose the node with minimum distance.

6: if selected node=destination node then

7: Go to step 13

8: else if energy of selected node is greater than threshold then

9: Repeat step 3 for selected node

10: else

11: Backtrack to step 5 and choose another node from remaining set of nodes.

12: Repeat step 3 for this selected node

13: end if

14: end for

15: End

The threshold value should be close to half of full scale energy. So, $4 \mathrm{~J}$ has been taken as threshold as it is close to half of $10 \mathrm{~J}$ that is $5 \mathrm{~J}$.

\section{PERFORMANCE EVALUATION}

\subsection{Simulation Setup}

MATLAB software has been used for the simulations of proposed algorithm due to its ease of node deployment and network set up. It has numerous built-in commands and math functions that help in mathematical calculations, generating plots and implementing algorithms. With the help of MATLAB 2013a, critical analysis of results is achieved.

500 nodes have been randomly deployed. Out of these nodes, 20 nodes have been randomly taken whose energy is set equal to $4 \mathrm{~J}$. The rest of the nodes have been provided $10 \mathrm{~J}$ of energy. The threshold value of energy is set for all the nodes (4J). Radio Energy Dissipation Model has been used by setting electronic energy per joule $=50 \mathrm{~nJ} / \mathrm{bit}$ and amplification energy $=10 \mathrm{pJ} / \mathrm{bit}$ square meter to calculate the remaining energy of nodes after these broadcast packets to their neighbors. The simulation is also done by taking different packet sizes.

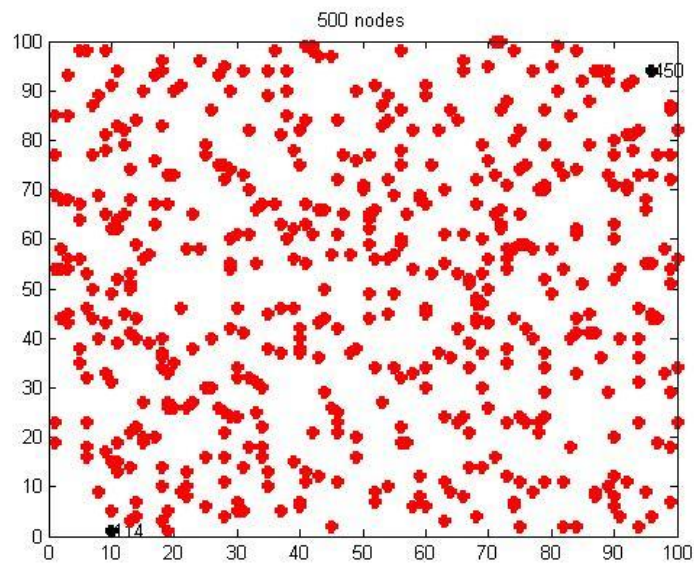

Fig 2: 500 nodes randomly deployed

Table 1: simulation Parameters

\begin{tabular}{|c|c|c|}
\hline $\begin{array}{c}\text { Sr. } \\
\text { No. }\end{array}$ & Parameters & Value \\
\hline 1 & Channel & Wireless \\
\hline 2 & Simulation Area & $100 * 100$ \\
\hline 3 & No. of nodes & 500 \\
\hline 4 & Packet size & $200,400,600,800,1000$ \\
\hline 6 & Threshold & $4 J$ \\
\hline 7 & Simulator & Matlab R2013a \\
\hline
\end{tabular}

\subsection{Simulation Results and Analysis}

\subsubsection{Analysis of RREP Phase of Nodes}

The node at the origin and extreme end are selected as source and destination respectively (say 114 and 450 respectively). Source and destination are represented by blue color and the remaining nodes by red color. The acknowledgement path followed for RREP phase has been shown with 'green' line. 
'Before' represents the graphs for AODV-TP protocol and 'After' represents the graphs for the modified AODV-TP protocol.
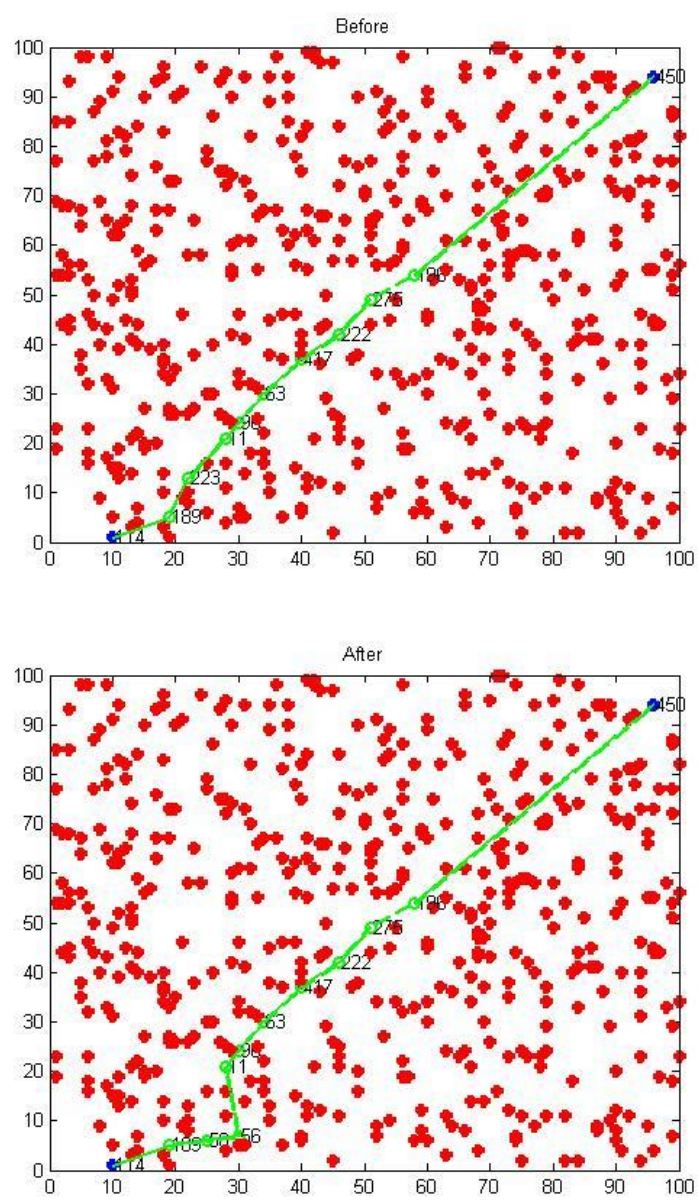

Fig 3: RREP phase of 500 nodes

\subsubsection{Energy Comparison}

It is observed that there is difference in the average remaining energy of the protocols with variation in packet size.

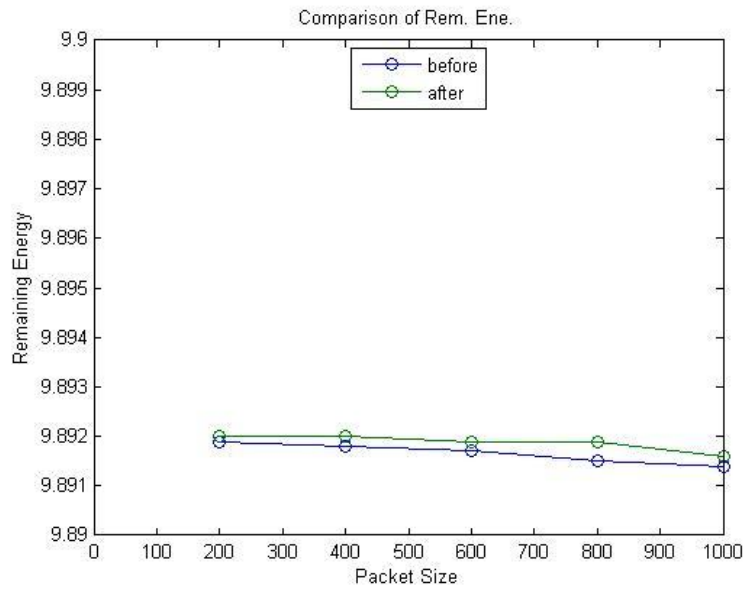

Fig 4: Comparison of remaining energy
The modified AODV-TP turns out to be more power efficient protocol than AODV-TP because in modified ASODV-TP only few nodes are involved in communication where as in AODV-TP all the neighbor nodes are involved in communication.

\subsubsection{Delay Comparison}

The time required for packets to travel from a specific source to a specific destination and back again is calculated.

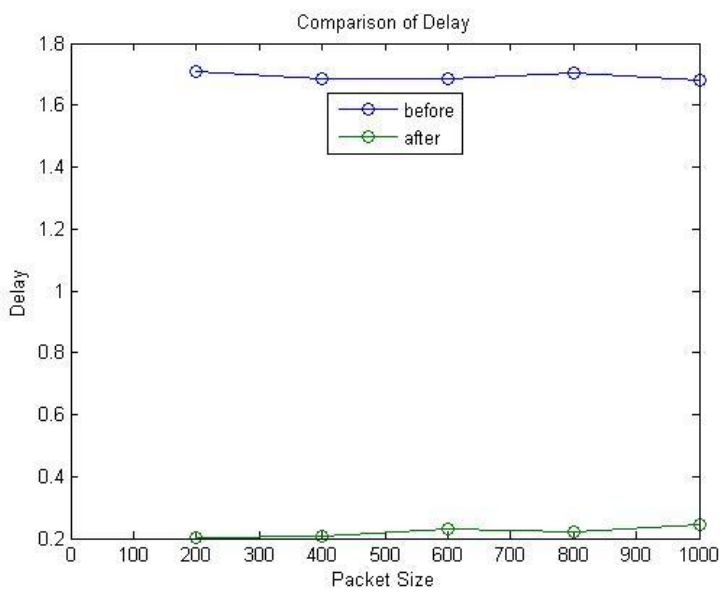

Fig 5: Comparison of delay

It is observed that the delay in modified AODV-TP is less than AODV-TP with variation in packet size because of the same reason as described in previous section.

\subsubsection{Routing Overhead Comparison}

Routing overhead is related to the total number of routing packets transmitted during simulation. It measures the scalability of a protocol, the degree to which it will function in congested or low bandwidth environments.

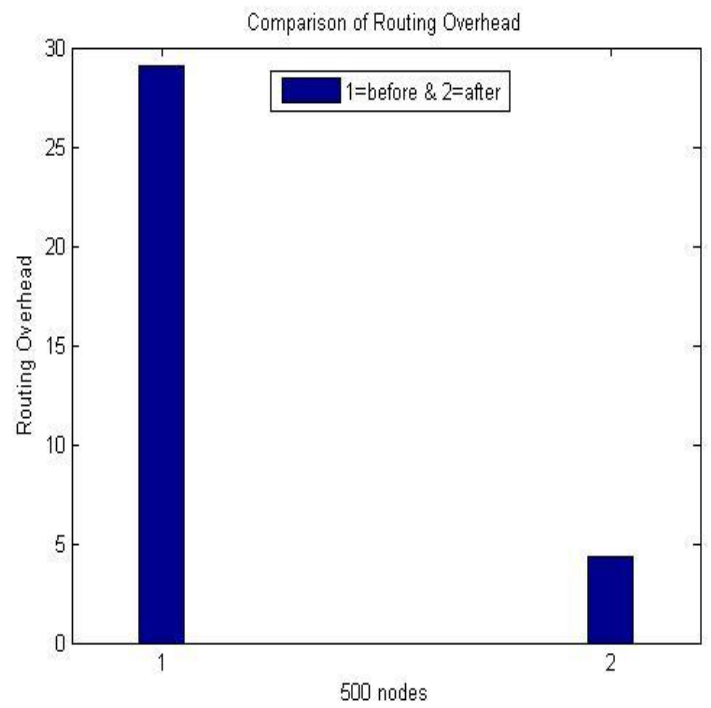

Fig 6: Comaprison of routing overhead

The routing overhead in the modified AODV-TP protocol decreases to a much extent as it is 4.33 in this case. In AODV$\mathrm{TP}$, it was 29.12. Routing Overhead remains same for different number of nodes because packet size remains constant. 
Table 2: Results comparison

\begin{tabular}{|c|c|c|}
\hline Packet Size & $\begin{array}{l}\text { Remaining } \\
\operatorname{Energy}(J)\end{array}$ & $\begin{array}{r}\text { Delay } \\
\text { (sec) }\end{array}$ \\
\hline 200 & & \\
\hline Before & 9.8919 & 1.7091 \\
\hline After & 9.8920 & 0.2038 \\
\hline 400 & & \\
\hline Before & 9.8918 & 1.6857 \\
\hline After & 9.8920 & 0.2078 \\
\hline 600 & & \\
\hline Before & 9.8917 & 1.6861 \\
\hline After & 9.8919 & 0.2313 \\
\hline 800 & & \\
\hline Before & 9.8915 & 1.7021 \\
\hline After & 9.8919 & 0.2199 \\
\hline 1000 & & \\
\hline Before & 9.8914 & 1.6824 \\
\hline After & 9.8916 & 0.2458 \\
\hline
\end{tabular}

\section{CONCLUSION}

AODV is not suitable for WSN because it considers simple hop count metric to select the best path. AODV-TP chooses that path in which that node participates in communication whose energy is greater than threshold. In modified AODV$\mathrm{TP}$, besides the above condition only those nodes are chosen as neighbors of node during RREQ phase where $\mathrm{x}$ as well as $y$ coordinate of the neighbors are greater than that of the corresponding coordinates of the source node. The comparison is done between the two in terms of routing overhead, average remaining energy of nodes and delay with variation in packet size. It is observed that modified AODVTP protocol is better in terms of power conservation. Also, the routing overhead and delay decreases in modified AODV-TP.

\section{FUTURE WORK}

Location based energy aware routing method- Location Aided Energy Efficient Routing (LAEER) protocol can be used for energy reduction in the network. LAEER protocol performs better than AODV in terms of routing overhead, average endto-end delay and average energy consumption in the network.
The simulations are required to be done for other parameters such as link capacity combined with the route selection logic to improve overall QoS of wireless network. AODV protocol can be simulated in IEEE 802.15.4 or Zigbee, analyzed using above performance metrics and its performance can be compared with IEEE 802.11. The future work can also be carried out to save energy of nodes when these are in idle mode.

\section{REFERENCES}

[1] A. Mainwaring, D. Culler, J. Polastre, R. Szewczyk, and J.Anderson, "Wireless sensor networks for habitat monitoring," ACM International Workshop Wireless Sensor Network Application, 2002, pp. 88-97

[2] S. Kim, S. Pakzad, D. Culler, J. Demmel, G. Fenves,S.Glaser, and M. Turon, "Health monitoring of civil infrastructures using wireless sensor networks," 6th International Conference on Information Procesing in Sensor Networks, New York, 2007, pp. 254-263.

[3] I. Stoianov, L. Nachman, S. Madden, and T. Tokmouline, "Pipenet: A wireless sensor network for pipeline monitoring," 6th International Conference on Information Procesing in Sensor Networks, New York, 2007, pp. 264-273

[4] A. Camilli, C. E. Cugnasca, A. M. Saraiva, A. R. Hirakawa, and P. L. P. Correa, "From wireless sensors to field mapping: Anatomy of an application for precision agriculture," Computer and Electronics in Agriculture, vol. 58, Issue 1, 2007, pp. 25-36

[5] C. Perkins, E. Royer, "Ad-Hoc On Demand Distance Vector Routing," the second IEEE workshops on Mobile Computer Systems and Applications, 1999, pp. 90-100

[6] Marina, M. K., Das S, R.; "On Demand multi-path Distance Vector Routing in Ad hoc network,"IEEE International Conference on Network Protocols, 2001, pp.14-23

[7] Seema Verma , Pinki Nayak and Rekha Agarwal "Energy Efficient Routing in Mobile Adhoc Networks based on AODV Protocol" International Journal of Computer Science Issues, Vol. 9, Issue 6, No 2, November 2012,pp.344-349

[8] P Samundiswary and S.R. Anandkumar," Throughput Analysis of Energy Aware Reactive Routing Protocol for Wireless Sensor Networks," International Journal of Soft Computing and Engineering), ISSN: 2231-2307, Volume2,Issue 1, March 2012, pp.497-500 